\title{
Regulating Speed and Generating Large Speed Transitions in a Neuromuscular Human Walking Model
}

\author{
Seungmoon Song and Hartmut Geyer
}

\begin{abstract}
Although current humanoid controllers can rely on inverse kinematics or dynamics of the full humanoid system, powered prosthetic legs or assistive devices cannot, because they do not have access to the full states of the human system. This limitation creates the need for alternative control strategies. One strategy is to embed fundamental knowledge about legged dynamics and control in local feedback. In a previous paper, we have developed a control model of human locomotion which relies mostly on local feedback. The model can robustly walk at normal walking speeds. Here we extend this model to adapt to a wide range of walking speeds and to generate corresponding speed transitions. We use optimization of the model's control parameters and find key parameters responsible for steady walking between $0.8 \mathrm{~ms}^{-1}$ and $1.8 \mathrm{~ms}^{-1}$, covering the range of speed at which humans normally walk. Using these parameters, we demonstrate speed transitions between slow and fast walking. In addition, we discuss how the speed-dependent changes of the identified control parameters connect to biped walking dynamics, and suggest how these changes can be integrated in local feedback control.
\end{abstract}

\section{INTRODUCTION}

Although current humanoid controllers can rely on inverse kinematics or dynamics of the full humanoid system [1]-[3], powered assistive devices [4]-[8] cannot, because they do not have access to the full states of the human system. Controllers that use mostly local information are indispensable for these systems. In line with this requirement, neuroscientific experiments [9]-[11] and simulation models [12]-[15] have shown that in animals and humans motor control of legged locomotion can largely rely on local sensory feedback control. For instance, decerebrate cats who have no connection between the central processing in the brain and the muscles in the legs, can walk and run on treadmills autonomously adapting to locomotion speed [16].

In a previous work [17], we have developed a neuromuscular model of human locomotion which uses mostly local sensory feedback to control walking. Based on this musclereflex control, the model adapts its locomotion pattern to variable ground including up and down slopes. Although this neuromuscular control promises novel control strategies for powered prosthetic legs [5], [6], the model's control requires several extensions to render it viable for complex robotic systems. First, the model is planar and does not account for frontal plane motion. Second, the model has been tuned for a single walking speed of $1.3 \mathrm{~ms}^{-1}$ equal to the

This work is sponsored by the DARPA Maximum Mobility and Manipulation Program under contract W911NR-11-1-0098.

$\mathrm{S}$. Song and $\mathrm{H}$. Geyer are with the Robotics Institute, Carnegie Mellon University, 5000 Forbes Avenue, Pittsburgh, PA 15213, USA. \{smsong, hgeyer\}@ cmu.edu preferred walking speed in humans; it currently does not have a controller that regulate different walking speeds.

In this paper, we focus on extending the neuromuscular control to adapt to a wide range of walking speeds and to generate corresponding speed transitions. Humans normally walk at speeds ranging from about $0.8 \mathrm{~ms}^{-1}$ to $1.8 \mathrm{~ms}^{-1}$ [18]. We first optimize for this range of speeds over all control parameters of the current neuromuscular model using the covariance matrix adaptation evolution strategy (CMAES) [19] (section II), and find nine key parameters for steady state walking at different speeds (section III). The nine parameters fall into three classes which account for trunk balance, stance behavior, and swing generation. In a second step, we optimize over these nine parameters to study which of these classes are critical to generate speed transitions between slow and fast walking (section IV). Our results show that to control large speed transitions (between $0.8 \mathrm{~ms}^{-1}$ and $1.8 \mathrm{~ms}^{-1}$ ) about two to five steps are required and all three classes contribute equally. We discuss preliminary architectures that embed the generation of speed transitions in the neuromuscular control.

\section{HUMAN MODEL AND ITS OPTIMIZATION}

In previous work, we have identified a forward-dynamic human model of neuromuscular control that generates steady-state walking behavior with human-like kinematics, kinetics, and muscle activations (Fig. 1). (Details of this model can be found in [17]; we here summarize its main components.) The model represents the human musculoskeletal system as a planar, seven segment system modeling the trunk as well as the thighs, shanks and feet. The segments are connected by revolute joints forming the hip, knee and ankle. The joints are actuated by seven Hill-type muscle models per leg, representing major leg muscles in human walking (soleus, SOL; gastrocnemius, GAS; tibialis anterior, TA; vastii group, VAS; hamstring group, HAM; gluteus maximus, GLU; and grouped hip flexors, HFL; Fig. 1). The muscle models consist of contractile elements, who take muscle stimulation signals $S_{m}$ from $0 \%$ to $100 \%$ as input, combined with series and parallel elasticities. Each muscle's force translates into a joint torque contribution $\tau_{m, j}=F_{m} \cdot r_{m}\left(\varphi_{j}\right)$ at the joints $j$ it spans, using variable moment arms $r_{m}\left(\varphi_{j}\right)$ that mimic the physiological moment arms observed for these muscles and joints.

The muscle stimulations $S_{m}$ are generated by the model's sensory feedback control. The control consists of separate stance and swing phase muscle reflexes which are based on 


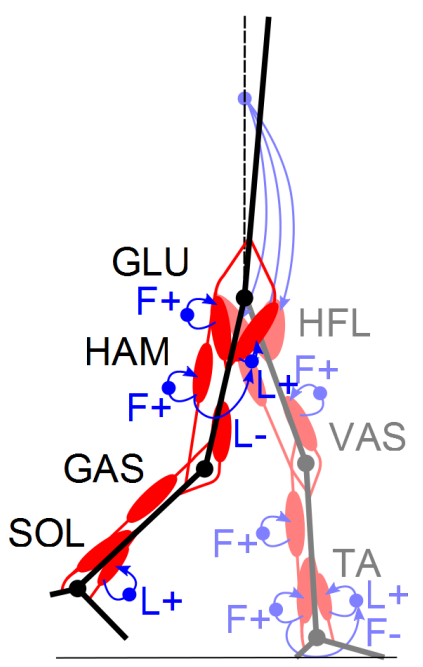

Fig. 1. Neuromuscular human walking model. The model has seven segments driven by 14 muscles. Segment masses and inertias reflect human data. Muscles are modeled as Hill-type muscles with force-length and force-velocity relationships, and include series and parallel springs. The control is purely reflexive. It does not require central pattern generators or precomputed joint trajectories. Key muscle reflexes active in swing (shown for left leg) are the positive length feedback $(\mathrm{L}+)$ of tibialis anterior (TA) lifting the foot, $\mathrm{L}+$ of the hip flexor and its suppression by positive force feedback $(F+)$ of the biarticular hamstring (HAM) and the glutei (GLU). Key reflexes in stance (right leg) are $F+$ of the leg extensors soleus (SOL), gastrocmenius (GAS) and vastii (VAS), negative force feedback $(F-)$ from SOL to TA suppressing the TA L+, and the trunk balance control around a reference lean from the vertical that activates either GLU and HAM or HFL based on a proportional-derivative signal of the forward lean.

sensory signals measuring the muscle state (mostly homonymous, positive force or length feedbacks, $F+$ or $L+$, Fig. 1). To reflect neural transport delays, these signals are timedelayed, as well as gained, and fed back into sum blocks that model alpha motoneurons and produce the muscle stimulations. The sensory feedbacks used in the model have been synthesized element by element from translating a bipedal spring-mass model [20] into an articulated one, embedding key principles of legged dynamics and control in sensory feedback control. (See [17] for details on this model. Note that in the current implementation, this model has an arched foot to resemble the human triangular foot geometry. The arched foot's weight and inertia match that of the rigid foot segment reported in [17]).

While this model generates human-like walking and tolerate ground disturbances, it does not have an explicit controller for speed changes. To identify such a controller, we use control parameter optimization based on the CMA-ES [19]. Our general implementation of the CMA-ES samples a population of 16 solutions from the space of control parameters that we consider according to a multivariate normal distribution, evaluates these solutions based on a cost function, and uses the 8 solutions with the lowest cost to reshape the covariance matrix of the normal distribution. For each optimization trial, we optimize over 300 generations. In a first optimization stage, we identify the key control parameters for steady state walking at different speeds from all control parameters that the model has (section III). In the

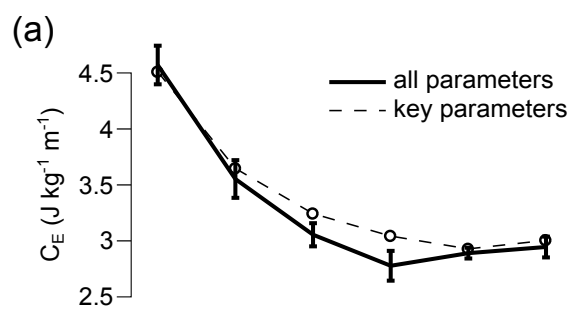

(b)

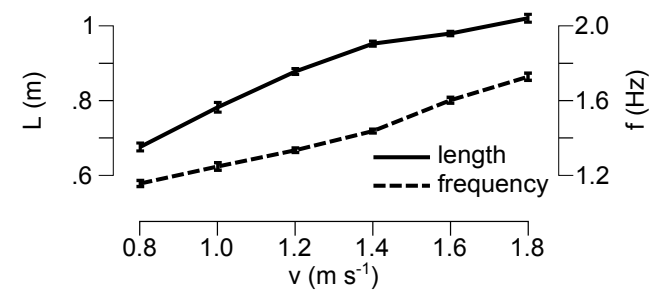

Fig. 2. Optimization of steady walking at different speeds in the model Shown are (a) mean \pm s.d. for the optimization results of the energetic cost with all the parameters (solid line, $n=5$ ) and only nine key parameters (dotted line, $n=1$ ), and (b) the resulting step length and frequency.

second, we optimize over the identified key parameters to elicit speed transitions and understand the underlying control strategies (section IV). Between these two optimization stages the actual cost function and optimization trial setup change as will be explained in sections III and IV.

\section{KEY CONTROL PARAMETERS FOR STEADY WALKING AT DIFFERENT SPEEDS}

\section{A. Steady Walking Optimization}

We divide the speed range from slow to fast walking into six levels ranging from $0.8 \mathrm{~ms}^{-1}$ to $1.8 \mathrm{~ms}^{-1}$ with $0.2 \mathrm{~ms}^{-1}$ increments. For each target speed, optimization trials start from steady walking at $1.3 \mathrm{~ms}^{-1}$ (for which we have hand-tuned control parameters), alter the full set of control parameters once, wait until the model again settles into steady walking, and then evaluate the cost function

$$
J=10\left|\dot{x}_{a v g}-\dot{x}_{t g t}\right|+P+C_{E},
$$

where $\dot{x}_{t g t}$ and $\dot{x}_{a v g}$ are the target speed and the average walking speed, $P$ is a pain term that accounts for joint overextensions, and $C_{E}$ is the energetic cost. We calculate $P$ as the sum of torques created by the soft mechanical joint limits in the neuromuscular model, which engage outside of the normal joint ranges $\left(70^{\circ}<\varphi_{a}<130^{\circ}, \varphi_{k}<175^{\circ}\right.$, and $\varphi_{h}<230^{\circ}$ for the ankle, knee and hip, respectively [17]). We compute the energetic cost as $C_{E}=E_{M} /\left(m \cdot x_{d}\right)$, where $m$ is the body mass, $x_{d}$ is the distance traveled, and $E_{M}$ is the total metabolic energy. $E_{M}$ is estimated as the sum of metabolic energies expended by all muscles, using the muscle energy model of [21]. We assume that an individual simulation has achieved steady walking as soon as the Cartesian positions of the joints do not change significantly relative to the touch-down position between subsequent heelstrikes (sum of differences $\leq 3 \mathrm{~cm}$ proved a reliable indicator of steady walking in extensive test simulations; note that 

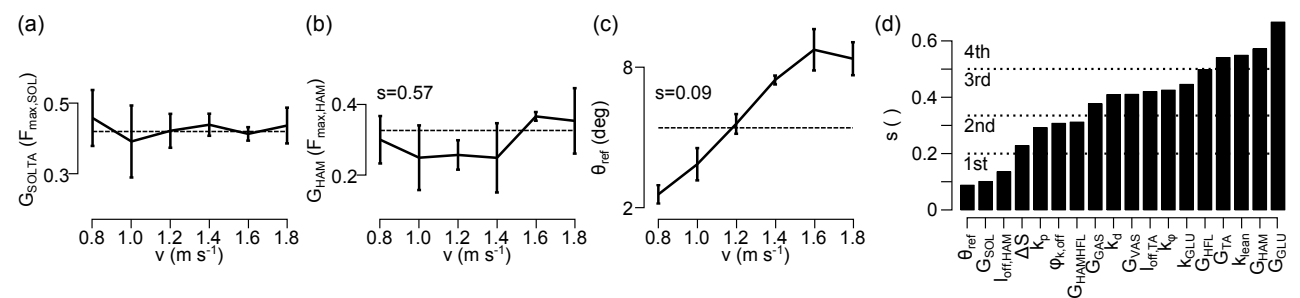

Fig. 3. Control parameter speed trends. For the full parameter optimization, the mean \pm s.d. $(n=5)$ are shown for (a) $G_{S O L T A}$, (b) $G_{H A M}$, and (c) $\theta_{\text {ref }}$. Dotted lines show the value which deviates least from the mean \pm s.d. $G_{S O L T A}$ has no trend, $G_{H A M}$ has a weak trend, and $\theta_{r e f}$ shows a clear trend. In (d), all parameters with a trend are sorted according to their trend value s. Small values indicate strong trends.

if no steady state is achieved, the simulation is repeated with a sample redrawn from the search space). For the three subsequent steady-state gait cycles, we compute the average walking speed, pain and energetic cost to determine the cost function $J$ (Eq. 1). To reduce the effect of local minima, we repeat the optimization five times for each target speed.

The cost function guarantees that the optimization seeks walking solutions which achieve the target speeds while avoiding unphysiological joint over-extensions and minimizing energetic effort. Both constraints are common in gait optimization to identify human locomotion patterns [22].

\section{B. Key Control Parameters}

The optimization repeatedly finds steady walking for all six target speeds from $0.8 \mathrm{~ms}^{-1}$ to $1.8 \mathrm{~ms}^{-1}$ with gait characteristics similar to those of humans. Figure 2 shows the resulting energetic cost (mean \pm s.d. over the five repetitions) as well as the step length $L$ and step frequency $f$ as two characteristic, speed-dependent parameters of human locomotion. The identified walking solutions show qualitative and quantitative agreement with human experimental data. The energetic cost shows the characteristic minimum of about $3 \mathrm{Jkg}^{-1} \mathrm{~m}^{-1}$ around normal speeds of $1.2 \mathrm{~ms}^{-1}$ to $1.4 \mathrm{~ms}^{-1}$ [23] (Fig. 2a), and $L$ and $f$ increase with speed $v=L f$ from $0.7 \mathrm{~m}$ to $1 \mathrm{~m}$ and $1.2 \mathrm{~Hz}$ to $1.8 \mathrm{~Hz}$, respectively, where the change of step length contributes more to speed increase at lower walking speeds and vice versa [24] (Fig. 2b).

To extract key control parameters that influence walking speed, we classify the identified control parameter changes (Fig. 3). We first discard all parameters that do not show a trend with speed. We assume that no trend exists for a parameter if a single value can be found (indicated as horizontal line in figure 3a) that lies within the identified parameter range (mean \pm s.d.) at all speeds, where the means and standard deviations are taken over the control parameter solutions that the five repetitions of the optimization found.

In a second step, we select only those parameters with a clear trend. For an indicator of the trend clarity, we compare the average magnitude of the standard deviations to difference in the smallest and largest mean parameter values, $s=s t d_{\text {avg }} /\left(\right.$ max $\left._{\text {mean }}-\min _{\text {mean }}\right)$. Figure $3 \mathrm{~b}$ and $3 \mathrm{c}$ show two examples for control parameters that both have a trend but different values of $\mathrm{s}$ with the latter parameter showing a much clearer trend than the former. Figure 3 lists all the 18 parameters that show a trend sorted by their $\mathrm{s}$ value. Several groups of parameters exist that have similar $\mathrm{s}$ values. We decide to drop control parameters with $s>0.33$, focusing on the first two groups $\left(\theta_{r e f}, G_{S O L}, l_{o f f, H A M}, \Delta S, k_{p}\right.$, $\varphi_{k, o f f}$, and $\left.G_{H A M H F L}\right)$.

In addition, we include two more control parameters with weaker trends because they are closely related to the seven selected ones. We include the d-gain $k_{d}$ of the trunk control because it defines the trunk balance together with $\theta_{\text {ref }}$, the trunk reference lean, and $k_{p}$, the p-gain of the trunk control. Similarly, we include the gain $k_{\varphi}$ that suppresses the VAS activity to prevent knee overextension together with the selected parameter $\varphi_{k, o f f}$, the start value of this VAS suppression control.

The resulting nine control parameters and their trends with walking speed are shown in Fig. 4. To ensure that we do not miss out on critical control parameters, we repeat the optimizations for all target speeds with the selected nine parameters while keeping the remaining 21 parameters constant. The values for these constants are set to $\left(\min _{\text {mean }+s t d}+\max _{\text {mean-std }}\right) / 2$, which deviates least from all mean \pm s.d. (dotted lines in Fig. 3a,b). The results show that the optimizations over the nine key control parameters find similar walking solutions with consistent parameter trends (dotted lines in Figs. 2 and 4).

\section{Parameter Interpretation}

The identified control parameters fall into three categories centered around trunk balance, swing leg motion, and stance leg behavior. The trunk parameters $\theta_{r e f}, k_{p}$ and $k_{d}$ influence the muscle stimulations that the hip flexor (HFL) and extensor muscles (GLU, HAM) get. This muscle stimulation is determined mainly by the proportional-derivative terms $k_{p}\left(\theta-\theta_{\text {ref }}\right)+k_{d} \dot{\theta}$, where $\theta$ and $\dot{\theta}$ are time delayed $(\Delta t=$ $5 \mathrm{~ms}$ ) signals of the trunk angle and angular velocity. If the sum is positive, the terms add to the stimulation of the hip extensors GLU and HAM; for negative values, the amount adds to the stimulation of the hip flexor HFL. The trends in figure 4a suggest that trunk forward lean is a major contributor to fast walking.

Intuition about the segment dynamics helps to understand the contribution of trunk lean to fast walking (Fig. 5a). Forward propulsion in legged locomotion can be created either by lengthening the leg after midstance or by rotating it about the hip. Hip rotation requires extension moments at that joint which accelerate the trunk backward. Trunk 


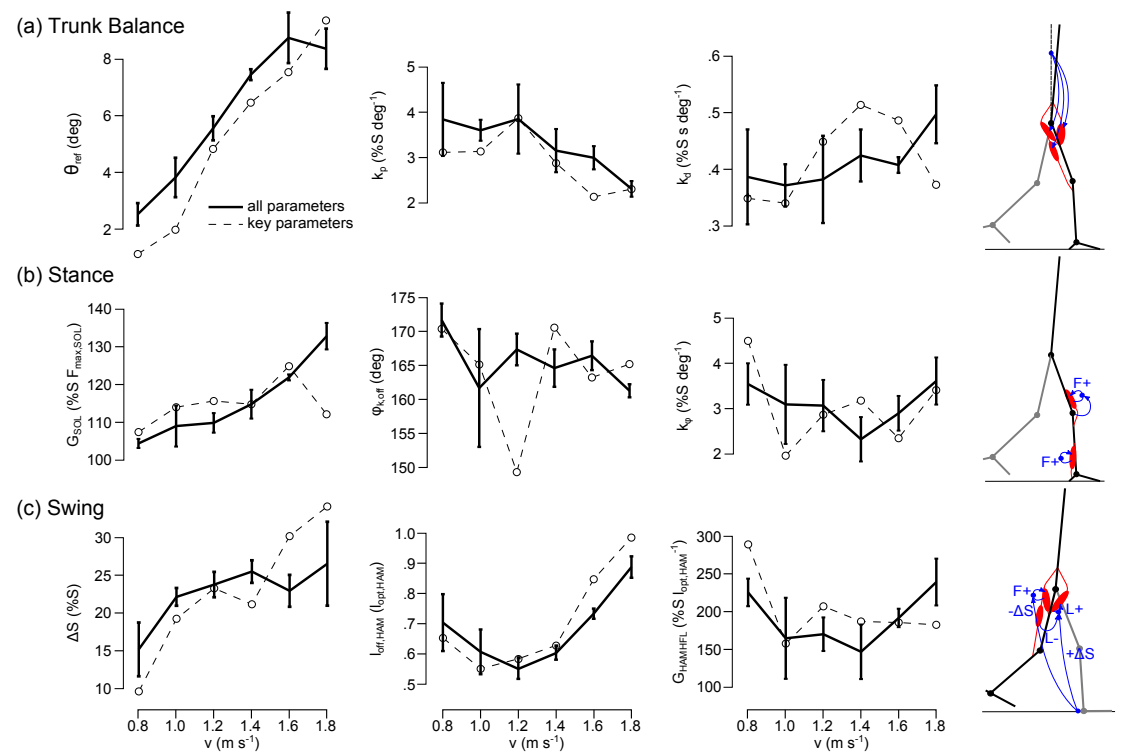

Fig. 4. Trends of the nine key control parameters for steady walking. Parameters are categorized by function into (a) trunk balance, (b) stance behavior, and (c) swing generation. Values are shown for the full optimization with all parameters (mean \pm s.d., $n=5$, solid line) and for the repeated optimization with only these nine key parameters (dotted line, $n=1$ ). Panels on the right indicate corresponding feedback control paths.

forward lean compensates for these moments as gravity and leg impacts create counter moments when the trunk's center of mass is located forward. As a result, faster walking can be generated by increasing hip extension moments during stance if the trunk leans more forward.

Although simulation studies on limit cycle walking robots have identified that, for controlling speed, lengthening the leg consumes more energy than leaning the trunk [25], this strategy creates energy efficient speed changes as well in our model (Fig. 4b). The identified stance parameter $G_{S O L}$ influences ankle push-off. The stimulation of the major ankle extensor muscle SOL is generated by positive feedback of the muscle force, $S_{S O L}=S_{0}+G_{S O L} F_{S O L}$, where $F_{S O L}$ is the time delayed ( $\Delta t=20 \mathrm{~ms})$ muscle force signal, $S_{0}$ is a small stimulation offset of $1 \%$, and $G_{S O L}$ is the feedback gain. Increasing the feedback gain results in a faster buildup of ankle extension torque, which increases the push-off toward the end of the stance.

The other two stance control parameters, $k_{\varphi}$ and $\varphi_{k, \text { off }}$, belong to a proportional control that prevents knee overextension by suppressing the major knee extensor muscle VAS according to $-k_{\varphi}\left(\varphi_{k}-\varphi_{k, o f f}\right)\left(\dot{\varphi}_{k}>0\right)$, where $\varphi_{k}$ and $\dot{\varphi}$ are time delayed $(\Delta t=10 \mathrm{~ms})$ sensory signals of the knee angle and angular velocity, and $\varphi_{k, o f f}$ is a threshold below which no signal is generated. While the actual trend of this control with speed is less clear, the increased hip and ankle extension torques due to trunk lean and push-off tend to overextend the knee (Fig. 5b), which the suppression of VAS can compensate.

Finally, the three swing-leg control parameters $\Delta S$, $l_{O f f, H A M}$ and $G_{H A M H F L}$ influence swing initiation and termination (Fig. 4c). In the neuromuscular model, swing is initiated during the double support phase in which the hip muscles receive a stimulation offset that increases the activity of HFL by $\Delta S$ and decreases that of GLU by the same amount. The net effect is a forward propulsion of the swing leg that, due to passive inertial coupling, also tends to flex the knee (Fig. 5c). Thus increasing the single parameter $\Delta S$ suffices to account for accelerated swing motions in fast walking.

The contribution of the other two swing control parameters is less clear. Although HFL activity continues during swing, it is modulated by feedback from other muscles. In particular, the HFL stimulation is reduced by a negative feedback of the HAM position, $G_{H A M H F L}\left[l_{C E, H A M}-l_{o f f, H A M}\right]$, where $l_{C E, H A M}$ is the time delayed $(\Delta t=5 \mathrm{~ms})$ signal of HAM's contractile element length, $l_{\text {of } f, H A M}$ represents the signal threshold of the HAM muscle spindle sensors, and $G_{H A M H F L}$ is the feedback gain. This feedback contributes to decelerating the thigh in forward motion, allowing the shank to overtake the thigh and prepare the leg for landing. The optimization finds this feedback shifted toward larger threshold values for faster walking above $1.2 \mathrm{~ms}^{-1}$. This shift compensates for the shift in the coordinate system of the HAM relative to the world frame that the trunk forward lean introduces. Why this feedback also shifts its threshold value toward slow speeds below $1.2 \mathrm{~ms}^{-1}$ is not clear and requires further analysis.

\section{CONTROLLED SPEED TRANSITIONS}

The neuromuscular model is robust enough that speed changes up to $\pm 0.2 \mathrm{~ms}^{-1}$ can be achieved by simply adjusting the key control parameters for steady walking according to their trends (Fig. 4). However, the previous optimization did not account for generating speed transitions and this simple adjustment strategy does not work for larger speed transitions in general. In this section, we focus on the extreme cases of speed transitions between $0.8 \mathrm{~ms}^{-1}$ and $1.8 \mathrm{~ms}^{-1}$ by 
(a)

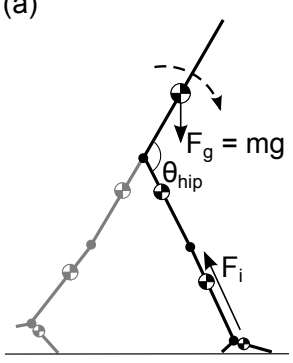

(b)

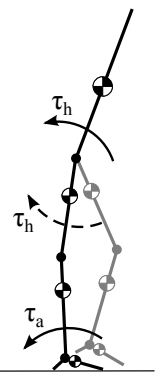

(c)

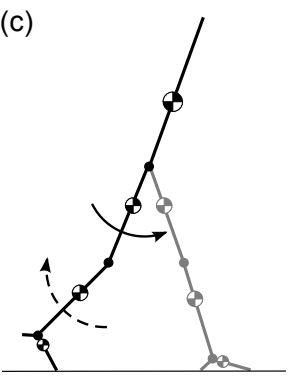

Fig. 5. Segment dynamics in three phases of walking including (a) leg loading, (b) midstance, and (c) swing initiation. Solid arrows denote active torques exerted muscle. dashed arrows mark reaction torques. $F_{g}$ and $F_{i}$ indicate trunk weight and impact force, $\theta_{h i p}$ is the hip angle and $\tau_{h / a}$ represents the active hip/ankle torque.

adopting temporary parameter changes during the transition phase.

\section{A. Speed Transition Optimization}

To find the temporary parameter changes that can generate large speed transitions from $0.8 \mathrm{~ms}^{-1}$ to $1.8 \mathrm{~ms}^{-1}$ and back, we devise a second optimization that takes advantage of the identified steady walking solutions. The optimization is based on trials in which the model starts from one identified steady walking solution with the control parameters set for $0.8 \mathrm{~ms}^{-1}$ or $1.8 \mathrm{~ms}^{-1}$ and then enters a transition period $\Delta_{p c}$ at the beginning of which the key control parameters are changed to generate the speed transition. After the period $\Delta_{p c}$, the parameters are set for the steady walking solution with $1.8 \mathrm{~ms}^{-1}$ or $0.8 \mathrm{~ms}^{-1}$ (Fig. 6). The transition period $\Delta_{p c}$ is not fixed but part of the cost function,

$$
\begin{aligned}
J= & 10\left|\dot{x}_{a v g}-\dot{x}_{t g t}\right|+10 P_{\Delta s t}+C_{E, \Delta s t} \\
& +n_{s t e p, \Delta s t}+n_{s t e p, \Delta p c},
\end{aligned}
$$

where $\left|\dot{x}_{a v g}-\dot{x}_{t g t}\right|$ is the difference between target speed and average speed at steady walking after the speed transition, $P_{\Delta s t}$ and $C_{E, \Delta s t}$ account for the pain and energetic cost terms during transition, $n_{s t e p, \Delta p c}$ is the number of steps during which the transition parameters are used, and $n_{s t e p, \Delta s t}$ is the number of steps it takes to reach steady walking with the new target speed. The cost function induces speed transitions to the target speed while minimizing pain, energetic cost, and the number of transition steps.

\section{B. Speed Transitions between Slow and Fast Walking}

We repeated the optimization of large speed transitions five times for both slow to fast and fast to slow walking. In the first case, the best solution accelerated from $0.8 \mathrm{~ms}^{-1}$ to steady walking at $1.8 \mathrm{~ms}^{-1}$ within four strides; in the second case, the best solution takes only two strides to decelerate from $1.8 \mathrm{~ms}^{-1}$ down to $0.8 \mathrm{~ms}^{-1}$. In both cases, the transition period $\Delta_{p c}$ lasted only one stride. Because the transitions were generated between previously identified steady walking solutions, we can control succeeding speed transitions between fast and slow walking. For example, figure 6 shows snapshots of the neuromuscular model in
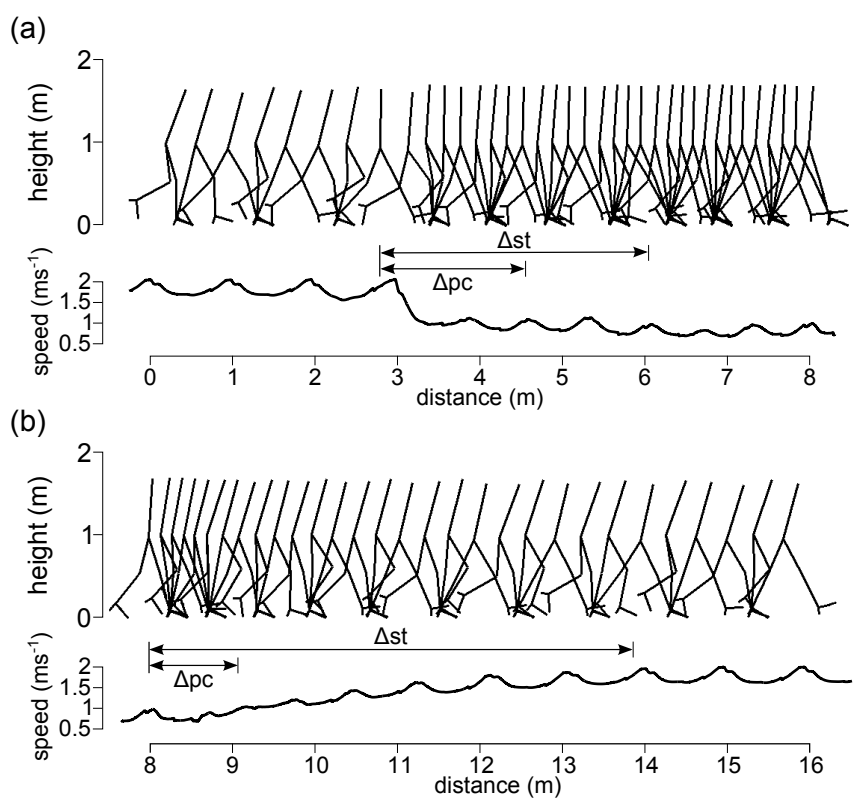

Fig. 6. Controlled speed transitions between slow and fast walking. (a) The neuromuscular model starts in steady walking with $1.8 \mathrm{~ms}^{-1}$, switches to slow walking at $x=3 \mathrm{~m}$, and reaches steady walking with $0.8 \mathrm{~ms}^{-1}$ after $\Delta_{s t}$. (b) Continued from (a), the return to fast walking with $1.8 \mathrm{~ms}^{-1}$ is shown. $\Delta_{p c}$ marks the transition interval with temporary parameter deviations. Snapshots are taken every $200 \mathrm{~ms}$.

200ms intervals during transitions from fast to slow walking and back.

\section{Potential Architecture for Speed Controller}

The optimization results further show that three key control parameters clearly deviate during transition, but only from slow to fast walking (Fig. 7). While the trunk reference lean $\theta_{\text {ref }}$ is similar during and after transition, the proportional and derivative gains, $k_{p}$ and $k_{d}$, of the trunk balance sharply increase during the transition stride (Fig. 7a). This increase is required to stop the trunk around its new reference lean after it gets accelerated forward due to the large jump in $\theta_{\text {ref }}$. The reverse change of $k_{p}$ and $k_{d}$ is not necessary during deceleration from fast to slow walking, because the trunk returns to a near upright position against the hip flexion torques created by the impact and gravity (Fig. 5a). The deviation suggests either a simple speed transition control that increases the trunk balance gains with the change $\Delta \theta_{\text {ref }}$ of the reference lean, or a more complex control that compensates for the asymmetric effects of leg impact and gravity on trunk balance. Both alternatives can be embedded with sensory feedbacks.

Connected to the large change in trunk lean, the gain $G_{S O L}$ of the positive force feedback of the SOL sharply drops during the transition stride from slow to fast walking. The drop reduces the ankle extensor moment, which ensures that knee overextension by exaggerated hip extension torques is avoided during the stopping of the trunk (Fig. 5b). An automated regulation of $G_{S O L}$ the SOL could originate directly from the control that increases the gains of the trunk balance. However, more careful analysis will be required 
(a) Trunk Balance
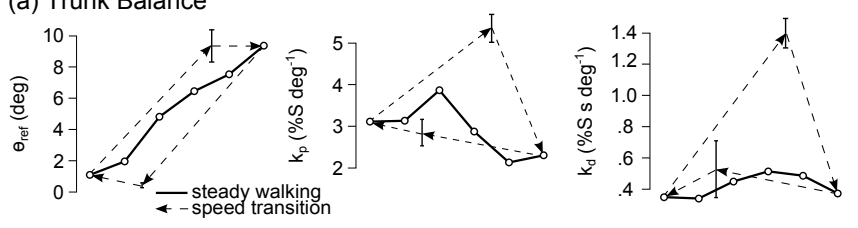

(c) Stance
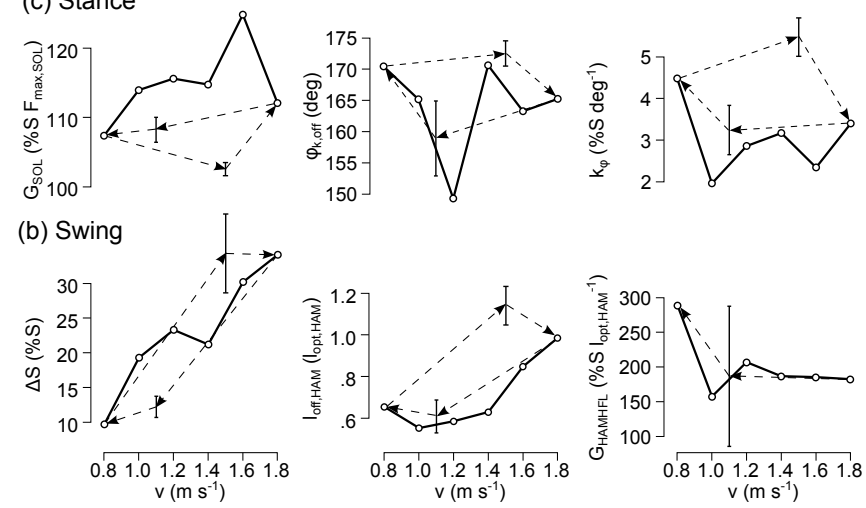

Fig. 7. Parameters deviations during speed transitions. Speed trends of the nine key parameters for steady walking (solid lines) (Fig. 4) are overlaid by the respective values during speed transitions (dashed lines, mean \pm s.d., $n=$ $5)$. The values for transitions $0.8 \mathrm{~ms}^{-1} \rightarrow 1.8 \mathrm{~ms}^{-1}$ and $1.8 \mathrm{~ms}^{-1} \rightarrow$ $0.8 \mathrm{~ms}^{-1}$ are plotted at $\mathrm{v}=1.1 \mathrm{~ms}^{-1}$ and $1.5 \mathrm{~ms}^{-1}$, respectively.

to clarify if such a control would compromise reactions to other disturbances and to explore alternative implementations. (Note that the other two stance parameters did not show a clear pattern that suggests controls.)

The three parameters of the swing control show either similar values during and after transition $(\Delta S)$ or do not show a clear pattern that suggests controls. In particular, $l_{C E, H A M}$ reached parameter values in the transition from slow to fast walking which cancelled the feedback output (no clear meaning for gain $G_{H A M H F L}$ ), and the gain $G_{H A M H F L}$ showed large variability in the transition from fast to slow walking.

\section{SUMMARY AND OUTLOOK}

Controllers that use only local information are indispensable for powered artificial legs and assistive devices because they do not have access to the full state of the human in the loop. We extended a neuromuscular model of human locomotion that uses only local sensory feedback to control walking, and identified key control parameters to automate large speed changes from slow to fast walking. Using these parameters, we could generate speed transitions between slow walking at $0.8 \mathrm{~ms}^{-1}$ and fast walking at $1.8 \mathrm{~ms}^{-1}$, well within the speed range that humans normally use [18]. In addition, we discussed how the trends of the identified control parameters connect to biped walking dynamics and made preliminary suggestions for automated integration of these changes in the sensory feedback control. We plan to realize these feedbacks in an integrated controller architecture in the model, and to extend the control to gait initiation and stopping. In addition, we plan to test our hypotheses about the sensory feedback changes during transition in experiments with humans.

\section{REFERENCES}

[1] R. L. Waters and S. Mulroy, "The energy expenditure of normal and pathological gait," Gait and Posture, vol. 9, pp. 207-231, 1999.

[2] B. Stephens and C. G. Atkeson, "Control of instantaneously coupled systems applied to humanoid walking," in IEEE-RAS Int. Conf. on Humanoid Robots, 2010, pp. 210-217.

[3] S. Song, Y. Ryoo, and D. Hong, "Development of an omnidirectional walking engine for full-sized lightweight humanoid robots," in Proc. ASME Int. Design Eng. Tech. Conf. Comp. Inform. Eng. Conf., 2011, in press.

[4] A. Chu, H. Kazerooni, and A. Zoss, "On the biomimetic design of the berkeley lower extremity exoskeleton (bleex)," in IEEE Int. Conf. Rob. Aut., 2005, pp. 4345-4352.

[5] M. F. Eilenberg, H. Geyer, and H. M. Herr, "Control of a powered ankle-foot prosthesis based on a neuromuscular model," IEEE Trans. Neural Syst. Rehabil. Eng., vol. 18, no. 2, pp. 164-173, 2010.

[6] J. Markowitz, P. Krishnaswamy, M. F. Eilenberg, K. Endo, C. Barnhart, and $\mathrm{H}$. Herr, "Speed adaptation in a powered transtibial prosthesis controlled with a neuromuscular model," Phil. Trans. R. Soc. B, vol. 366, pp. 1621-1631, 2011.

[7] A. M. Technologies, "Rewalk," 2011, http: / /www. argomedtec.com/products.asp.

[8] Honda, "Walking assist," 2011, http://corporate.honda.com/innovation/walk-assist/.

[9] G. Orlovsky, T. Deliagina, and S. Grillner, Neuronal Control of Locomotion: From Mollusc to Man. New York: Oxford Univ. Press, 1999.

[10] K. Pearson, O. Ekeberg, and A. Buschges, "Assessing sensory function in locomotor systems using neuro-mechanical simulations," Trends. Neurosci., vol. 29, no. 11, pp. 625-631, 2006.

[11] B. W. Verdaasdonk, H. F. J. M. Koopman, and F. C. T. V. der Helm, "Resonance tuning in a neuro-musculo-skeletal model of the forearm," Biol. Cybern., vol. 96, no. 2, pp. 165-180, 2007.

[12] G. Taga, "A model of the neuro-musculo-skeletal system for human locomotion. i. emergence of basic gait," Biol. Cybern., vol. 73, no. 2, pp. 97-111, 1995.

[13] N. Ogihara and N. Yamazaki, "Generation of human bipedal locomotion by a bio-mimetic neuro-musculo-skeletal model," Biol. Cybern., vol. 84, no. 1, pp. 1-11, 2001.

[14] M. Günther and H. Ruder, "Synthesis of two-dimensional human walking: A test of the $\lambda$-model," Biol. Cybern., vol. 89, pp. 89-106, 2003

[15] C. Paul, M. Bellotti, S. Jezernik, and A. Curt, "Development of a human neuro-musculo-skeletal model for investigation of spinal cord injury," Biol. Cybern., vol. 93, no. 3, pp. 153-170, 2005.

[16] M. L. Shik and G. N. Orlovskii, "Neuron responses of the "locomotor strip" in the lower brainstem of the cat," Neurophysiol., vol. 10, pp. 510-518, 1978.

[17] H. Geyer and H. M. Herr, "A muscle-reflex model that encodes principles of legged mechanics produces human walking dynamics and muscle activities," IEEE Trans. Neural Syst. Rehab. Eng., vol. 18, no. 3,2010

[18] M. P. Murray, L. A. Mollinger, G. M. Gardner, and S. B. Sepic, "Kinematic and emg patterns during slow free and fast walking," Journal of Orthopaedic Research, vol. 2, pp. 272-280, 1984.

[19] N. Hansen, "The cma evolution strategy: A comparing review," Towards a New Evolutionary Computation. Advances on Estimation of Distribution Algorithms. Springer, p. 75.102, 2006.

[20] H. Geyer, A. Seyfarth, and R. Blickhan, "Positive force feedback in bouncing gaits?" Proc. R. Soc. B, vol. 270, pp. 2173-2183, 2003.

[21] B. R. Umberger, K. G. Gerritsen, and P. E. Martin, "A model of human muscle energy expenditure," Comp. Meth. Biomech. Biomedic. Eng., vol. 6, no. 2, pp. 99-111, 2003.

[22] B. R. Umberger, "Stance and swing phase costs in human walking," J. R. Soc. Interface, vol. 7, no. 50, pp. 1329-1340, 2010.

[23] A. E. Minetti, J. Pinkerton, and P. Zamparo, "From bipedalism to bicyclism: evolution in energetics and biomechanics of historic bicycles," Proc. R. Soc. Lond. B, vol. 268, pp. 1351-1360, 2001.

[24] E. Hirasaki, S. T. Moore, T. Raphan, and B. Cohen, "Effects of walking velocity on vertical head and body movements during locomotion," Exp. Brain. Res., vol. 127, pp. 117-130, 1999.

[25] D. G. E. Hobbelen and M. Wisse, "Controlling the walking speed in limit cycle walking," Int. J. Robotics Research, vol. 27, no. 9, pp. 989-1005, 2008. 